\title{
Kinematic Basis of Emergent Energetics of Complex Dynamics
}

\author{
Hong QIAN, Yu-CHEN CHENG and YING-JEN YANG
}

Department of Applied Mathematics, University of Washington, Seattle, WA 98195-3925, USA

\begin{abstract}
PACS $02.50 . \mathrm{Ga}-$ Markov processes
PACS 05.70. Ln - statistical thermodynamics

PACS $05.40 . \mathrm{Ca}$ - fluctuation phenomena
\end{abstract}

\begin{abstract}
Stochastic kinematic description of a complex dynamics is shown to dictate an energetic and thermodynamic structure. An energy function $\varphi(\mathbf{x})$ emerges as the limit of the generalized, nonequilibrium free energy of a Markovian dynamics with vanishing fluctuations. In terms of the $\nabla \varphi$ and its orthogonal field $\gamma(\mathbf{x}) \perp \nabla \varphi$, a general vector field $\mathbf{b}(\mathbf{x})$ can be decomposed into $-\mathbf{D}(\mathbf{x}) \nabla \varphi+\gamma$, where $\nabla \cdot(\omega(\mathbf{x}) \gamma(\mathbf{x}))=$ $-\nabla \omega \mathbf{D}(\mathbf{x}) \nabla \varphi$. The matrix $\mathbf{D}(\mathbf{x})$ and scalar $\omega(\mathbf{x})$, two additional characteristics to the $\mathbf{b}(\mathbf{x})$ alone, represent the local geometry and density of states intrinsic to the statistical motion in the state space at $\mathbf{x} . \varphi(\mathbf{x})$ and $\omega(\mathbf{x})$ are interpreted as the emergent energy and degeneracy of the motion, with an energy balance equation $\mathrm{d} \varphi(\mathbf{x}(t)) / \mathrm{d} t=\gamma \mathbf{D}^{-1} \boldsymbol{\gamma}-\mathbf{b D}^{-1} \mathbf{b}$, reflecting the geometrical $\|\mathbf{D} \nabla \varphi\|^{2}+\|\boldsymbol{\gamma}\|^{2}=\|\mathbf{b}\|^{2}$. The partition function employed in statistical mechanics and J. W. Gibbs' method of ensemble change naturally arise; a fluctuation-dissipation theorem is established via the two leading-order asymptotics of entropy production as $\epsilon \rightarrow 0$. The present theory provides a mathematical basis for P. W. Anderson's emergent behavior in the hierarchical structure of complexity science.
\end{abstract}

Introduction. - Classical mechanics has traditionally been divided into kinematics and dynamics. The former gives the precise relationship between a mechanical motion $\mathbf{x}(t)$ and descriptions of the motion in terms of its velocity $\dot{\mathbf{x}}(t)$ and acceleration $\ddot{\mathbf{x}}(t)$ under geometric constraints, and the latter provides relationships between the motions and the concepts of mass, force, and mechanical energy. While the former is a part of calculus, the latter constitutes the core of the classical physics of motion. When a mechanical system contains a great rnany number of point masses such as atoms and molecules, the notions of heat and temperature, as a stochastic description of complex mechanical motions and kinetic energy, arise.

In classical, macroscopic chemical kinetics, a reaction in aqueous solution, say $A+B \rightarrow C$, is described by a rate process: $\mathrm{d} c_{A}(t) / \mathrm{d} t=-r(t)$, which should be identified as the chemical kinematics. It is again based on calculus which rigirously defines the concept of instantaneous rate of concentration change (fluxion). The functional relationship between $r(t)$ and the concentrations $c_{A}(t)$ and $c_{B}(t)$, known as a rate law, is analogous to the constitutive relations. One wellknown example of a rate law is the Guldberg-Waage mass action $r(t)=k c_{A}(t) c_{B}(t)$ where $k$ is a constant independent of $c_{A}$ and $c_{B}$. The notion of energy in chemical reactions, however, exists in the chemical thermodynamics of heterogeneous substants, a separate theory developed by J. W. Gibbs, who introdued the notion of Gibbs function and chemical potential as the energy and force that drives the chemical changes.

With the above understandings, therefore, it came as a surprise that a recent work [1,2] claims that the mathematical foundation of Gibbsian chemical thermodynamics, at a given temperature, needs only the mesoscopic stochastic kinematics, irrespective of any details of the mechanics of the atoms and molecules. In other words, the isothermal chemical thermodynamics of Gibbs is dictated purely by the kinematics via the mathematics of probability. The implication of this observation is conceptually sigificant: It implies in general a stochastic description of a complex dynamics has a "hidden" energetics that is already defined by mathematics! The present paper applies this novel idea, stochastic kinematics dictates energetics, to another general class of processes and further explore the idea: diffusion processes in continuous space $\mathbb{R}^{n}$ with continuous time $t \in \mathbb{R}$. Mathematical analysis again reveals a hidden energetic and thermodynamic structure that underlying the kinematics. The term "thermo" here does not imply heat; rather it means a stochastic description of a complex dynamics.

The concept of thermodynamic force was clearly articulated in the work of L. Onsager [3]. In chemical kinetics, it is widely accepted that entropic force is a legitimate description on par with mechanical force as a "cause" for an action [4, 5]. The Shannon entropy is computable in any statistical description of dynamics [6,7]. Actually, P. W. Anderson has stated that "[A]t each level of complexity entirely new properties appear, and the 
understanding of the new behaviors requires research which I think is as fundamental in its nature as any other." [8] In fact, he continued to provide a recipe for discovering an emergent law:

"It is only as the nucleus is considered to be a manybody system - in what is often called the $N \rightarrow \infty$ limit — that such [emergent] behavior is rigorously definable. ... Starting with the fundamental laws and a computer, we would have to do two impossible things - solve a problem with infinitely many bodies [e.g., zero fluctuations], and then apply the result to a finite system - before we synthesized this behavior."

As we shall see, the discovery of the hidden thermodynamic laws indeed involves taking the limit of noise, e.g., fluctuations, tending zero. Our result thus provides a clear understanding of why and how emergent thermodynamic behaviors, as statistical laws, can be independent of the underlying details.

We consider the general description of complex dynamics in terms of a stochastic Markov process $\mathbf{X}_{\epsilon}(t)$. Such a dynamics can be represented by its probability distribution $p_{\epsilon}(\mathbf{x}, t)$ that follows a Fokker-Planck equation (FPE) [9]

$$
\frac{\partial p_{\epsilon}}{\partial t}=-\nabla \cdot \mathbf{J}\left[p_{\epsilon}\right], \mathbf{J}\left[p_{\epsilon}\right] \equiv \mathbf{b}(\mathbf{x}) p_{\epsilon}-\epsilon \mathbf{D}(\mathbf{x}) \nabla p_{\epsilon} .
$$

Its trajectory is represented in terms of the solution to a Langevin type equation $\mathrm{dX}_{\epsilon}(t)=\tilde{\mathbf{b}}\left(\mathbf{X}_{\epsilon}\right) \mathrm{d} t+$ $\left[2 \epsilon \mathbf{D}\left(\mathbf{X}_{\epsilon}\right)\right]^{\frac{1}{2}} \mathrm{~d} \mathbf{B}(t)$, where $\mathbf{B}(t)$ is the standard multidimentional Brownian motion and $\tilde{\mathbf{b}}=\mathbf{b}+\nabla \cdot \epsilon \mathbf{D}$. The $\epsilon$ signifies a connection between deterministic and stochastic motions [10]: In the limit of $\epsilon \rightarrow 0$, the stochastic trajectory $\mathbf{X}_{\epsilon}(t) \rightarrow \hat{\mathbf{x}}(t)$ which satisties $\mathrm{d} \hat{\mathbf{x}}(t) / \mathrm{d} t=\mathbf{b}(\hat{\mathbf{x}}(t))$, and similarly $p_{\epsilon}(\mathbf{x}, t) \rightarrow$ $\delta(\mathbf{x}-\hat{\mathbf{x}}(t))$ if initial value $p_{\epsilon}(\mathbf{x}, 0)=\delta(\mathbf{x}-\hat{\mathbf{x}}(0))$.

We emphasize that a stochastic description of dynamics does not have any notion of "energy" and "thermodynamics" at the onset. This is what we meant by "kinematic description". As we shall show, however, in addition to the actual limit $\hat{\mathbf{x}}(t)$, a mathematico-thermodynamic structure also emerges in the process of taking $\epsilon \rightarrow 0$ : For a fixed $\epsilon$ and regarding the stochastic dynamics in Eq. (1), it is known that the relative entropy

$$
F\left[p_{\epsilon}(\mathbf{x}, t)\right] \equiv \int_{\mathbb{R}^{n}} p_{\epsilon}(\mathbf{x}, t) \ln \left(\frac{p_{\epsilon}(\mathbf{x}, t)}{\pi_{\epsilon}(\mathbf{x})}\right) \mathrm{d} \mathbf{x},
$$

has a paramount importance [11-[13], where the stationary solution to (1) $\pi_{\epsilon}(\mathbf{x})$ embodies the notion of an entropic force. It was discovered only recently that the $F$ satisfies a balance equation $\mathrm{d} F / \mathrm{d} t \equiv-f_{\mathrm{d}}(t)=Q_{\mathrm{hk}}(t)-e_{\mathrm{p}}(t)$ [14-17], with

$$
\begin{aligned}
f_{\mathrm{d}}\left[p_{\epsilon}\right] & =\int_{\mathbb{R}^{n}} \mathbf{J}\left[p_{\epsilon}\right] \cdot \nabla \ln \left(\frac{p_{\epsilon}}{\pi_{\epsilon}}\right) \mathrm{d} \mathbf{x} \\
Q_{\mathrm{hk}}\left[p_{\epsilon}\right] & =\int_{\mathbb{R}^{n}} \mathbf{J}\left[p_{\epsilon}\right] \cdot(\epsilon \mathbf{D})^{-1}(\mathbf{x}) \mathbf{J}\left[\pi_{\epsilon}\right] \pi_{\epsilon}^{-1} \mathrm{~d} \mathbf{x}, \\
e_{\mathrm{p}}\left[p_{\epsilon}\right] & =\int_{\mathbb{R}^{n}} \mathbf{J}\left[p_{\epsilon}\right] \cdot(\epsilon \mathbf{D})^{-1}(\mathbf{x}) \mathbf{J}\left[p_{\epsilon}\right] p_{\epsilon}^{-1} \mathrm{~d} \mathbf{x} .
\end{aligned}
$$

All three quantities in Eq. (3) are non-negative. This fact ensures the interpretation of $Q_{\mathrm{hk}}$ and $e_{\mathrm{p}}$ as the source and sink for the "free energy" of a system. The $F\left[p_{\epsilon}\right]$ is interpreted as a generalized, nonequilibrium free energy since $-\epsilon \int_{\mathbb{R}^{n}} p_{\epsilon} \ln \pi_{\epsilon} \mathrm{dx},-\int_{\mathbb{R}^{n}} p_{\epsilon} \ln p_{\epsilon} \mathrm{dx}$, and $\epsilon$ are analogous to "mean internal energy" $E$, entropy $S$, and temperature $T$, thus $F=E-T S$. See [18-20] for the relation between these average quantities and the trajectory-based stochastic thermodynamics, Jarzynski like equalities, and fluctuation theorems.

The key result of the present work is the physical interpretation of a set of three equations in Eqs. (12) putting together as a system. The three equations were known but not with the thermodynamic connections and the geometric interpretation shown in this letter. Eqs. 12a,b were in the mathematical work of Ventsel and Freidlin and the studies that followed [21-23]. (12k) has been derived in the work of Graham and Tél [24]. They focused on constructing asymptotic stationary probability distributions based on the three equations.

The present work illustrates a deep relation between this set of equations as a dynamic description and thermodynamic energetics, in the context of the recently developed stochastic thermodynamics. We regard Eq. (12) as an emergent energetics that accompanies the deterministic dynamics $\dot{\mathbf{x}}=\mathbf{b}(\mathbf{x})$. As a characterization of a complex behavior, the $\mathbf{b}(\mathbf{x})$ does not exist alone as a macroscopic description, it comes with additional information coded in $\mathbf{D}, \varphi$, and $\omega$, as thermodynamics. The Pythagorean relation (20) among the key quantities in (12) further suggests a geometric perspective to be explored.

We note the Eq. (12 a) is not a Helmoholtz (or Hodge) decomposition of a vector field since $\gamma(\mathbf{x})$ is not divergence free [25]. The motion following $\gamma(\mathbf{x})$ however, conserves the $\varphi(\mathbf{x})$ according to Eq. (12b). The divergence of $\gamma(\mathbf{x})$ is provided by the third equation $(12 \mathrm{k})$. The emergence of the $\omega(\mathrm{x})$ indicates the significance of a "local volume" of this $\varphi$-conservative motion [26]: This is called degeneracy in the classical statistical mechanical termonology. While the motion following $\gamma(\mathbf{x})$ can be complex, a proper statistical description, called physical measure, usually exists [27,28]. For more discussion of the thermodynamic meanings of dissipative vs. conservative motions, and their relation to trajectory-based entropy production, see [10, 25].

Emergent potential and $\varphi$-conservative motion $\gamma \cdot-\quad$ The mathematical theory of large deviations connects the stochastic dynamics with finite $\epsilon$ to the deterministic dynamics with $\epsilon=0$ $[30,35-39]$. It is a rigorous and complete asymptotic theory akin to the WKB ansatz:

$$
p_{\epsilon}(\mathbf{x}, t)=\exp \left[-\varphi^{\mathrm{td}}(\mathbf{x}, t) / \epsilon+o\left(\epsilon^{-1}\right)\right],
$$

in which $\varphi^{\mathrm{td}}(\mathbf{x}, t)$ is known as a time-dependent large deviation rate function. 11 Taking the Eq. (4) as given and recall that $p_{\epsilon}(\mathbf{x}, t) \rightarrow \delta(\mathbf{x}-\hat{\mathbf{x}}(t))$, then it is easy to see that in the limit of $\epsilon \rightarrow 0$,

$$
\epsilon F\left[p_{\epsilon}(\mathbf{x}, t)\right] \rightarrow \varphi^{\mathrm{ss}}(\hat{\mathbf{x}}(t)), \varphi^{\mathrm{ss}}(\mathbf{x})=-\lim _{\epsilon \rightarrow 0} \epsilon \ln \pi_{\epsilon}(\mathbf{x}) .
$$

${ }^{1}$ The $\varphi^{\mathrm{td}}(\mathbf{x}, t)$ satisfies a time-dependent equation of its own: $\partial \varphi^{\mathrm{td}}(\mathbf{x}, t) / \partial t=-\nabla \varphi^{\mathrm{td}}(\mathbf{x}, t) \cdot \boldsymbol{\gamma}^{\mathrm{td}}(\mathbf{x}, t)$, where $\boldsymbol{\gamma}^{\mathrm{td}}(\mathbf{x}, t) \equiv$ $\mathbf{D}(\mathbf{x}) \nabla \varphi^{\mathrm{td}}(\mathbf{x}, t)+\mathbf{b}(\mathbf{x})$. This is known as a Hamilton-Jacobi equation. 
Since $\mathrm{d} F / \mathrm{d} t=-f_{\mathrm{d}} \leq 0, F\left[p_{\epsilon}(\mathbf{x}, t)\right]$ is a monotonic nonincresing function of $t$. Consequently, Eq. (5) states that $\varphi^{\mathrm{ss}}(\hat{\mathbf{x}}(t))$ is a monotonic function of $t$, which implies that $\varphi^{\mathrm{ss}}(\mathbf{x})$ is an energy function of the dissipative dynamics $\hat{\mathbf{x}}(t)$, the solution to the deterministic equation $\mathrm{dx} / \mathrm{d} t=\mathbf{b}(\mathbf{x})$. We shall drop the superscript from the steady-state large deviation rate function $\varphi^{\mathrm{ss}}(\mathbf{x})$ from now on.

The connection between $F\left[p_{\epsilon}\right]$ and $\varphi(\mathbf{x})$ illustrates that the latter is an emergent energetic quantity in the limit of $\epsilon \rightarrow 0$. It provides a new derivation with thermodynamic insights of the mathematical result of Ventsel and Freidlin [21-23]. The relation firmly connects (mesoscopic) stochastic free energy $F\left[p_{\epsilon}\right]$ with (macroscopsic) deterministic pseudo-potential $\varphi(\mathbf{x})$; it validates the earlier interpretation of $-\epsilon \int_{\mathbb{R}^{n}} p_{\epsilon} \ln \pi_{\epsilon} \mathrm{d} x$ as a mean internal energy, with $\varphi(\mathbf{x})$ as the internal energy function of state $\mathrm{x} \in \mathbb{R}^{n}$.

We emphasize that the original $\mathbf{b}(\mathbf{x})$ in Eq. (1) is not a gradient field in general. In terms of the newfound $\varphi(\mathbf{x})$ and continue the WKB ansatz, one can express $\pi_{\epsilon}(\mathbf{x})=$ $\omega(\mathbf{x}) \exp [-\varphi(\mathbf{x}) / \epsilon+a \ln \epsilon+O(\epsilon)]$, in which $\ln \omega(\mathbf{x})$ is the next order to the leading two terms and $a \ln \epsilon$ arising from normalization fractor is independent of $\mathrm{x}$. Substituting this expression into the stationary FPE

$$
-\nabla \cdot \mathbf{J}\left[\pi_{\epsilon}(\mathbf{x})\right]=\nabla \cdot\left(\epsilon \mathbf{D}(\mathbf{x}) \nabla \pi_{\epsilon}(\mathbf{x})-\mathbf{b}(\mathbf{x}) \pi_{\epsilon}(\mathbf{x})\right)=0,
$$

one obtains

$$
\begin{gathered}
\epsilon^{-1} \omega(\mathbf{x}) \gamma(\mathbf{x}) \cdot \nabla \varphi-[\nabla \omega(\mathbf{x}) \cdot \mathbf{D}(\mathbf{x}) \nabla \varphi+\nabla \cdot(\omega(\mathbf{x}) \gamma(\mathbf{x}))] \\
+\epsilon \nabla \cdot(\mathbf{D}(\mathbf{x}) \nabla \omega(\mathbf{x}))+O(\epsilon)=0,
\end{gathered}
$$

in which $\gamma(\mathbf{x}) \equiv \mathbf{D}(\mathbf{x}) \nabla \varphi(\mathbf{x})+\mathbf{b}(\mathbf{x})$. Equating like order terms in Eq. (7), we have

$$
\nabla \varphi(\mathbf{x}) \cdot \gamma(\mathbf{x})=0, \forall \mathbf{x} \in \mathbb{R}^{n},
$$

the vector field $\gamma(\mathbf{x})$ is orthogonal to $\nabla \varphi(\mathbf{x})$, and [24]

$$
\nabla \cdot(\omega(\mathbf{x}) \gamma(\mathbf{x}))=-\nabla \omega(\mathbf{x}) \cdot \mathbf{D}(\mathbf{x}) \nabla \varphi(\mathbf{x}) .
$$

Actually, $\gamma_{\epsilon}(\mathbf{x}) \equiv \pi_{\epsilon}^{-1}(\mathbf{x}) \mathbf{J}\left[\pi_{\epsilon}(\mathbf{x})\right]$ has been identified as Onsager's thermodynamic flux [40], and $\gamma$ is its limit as $\epsilon \rightarrow 0$ :

$$
\lim _{\epsilon \rightarrow 0} \gamma_{\epsilon}(\mathbf{x})=\mathbf{b}(\mathbf{x})+\mathbf{D}(\mathbf{x}) \nabla \varphi(\mathbf{x}) \equiv \gamma(\mathbf{x}) .
$$

The motions following the vector field $\gamma(\mathbf{x})$ is restricted on the level set of $\varphi(\mathbf{x})$. This result also has a correspondence when $\epsilon$ is finite [41,44]: The stationary FPE can be re-written as

$$
\nabla \varphi_{\epsilon}(\mathbf{x}) \cdot \gamma_{\epsilon}(\mathbf{x})=\epsilon \nabla \cdot \gamma_{\epsilon}(\mathbf{x}),
$$

in which $\varphi_{\epsilon}(\mathbf{x}) \equiv-\epsilon \ln \pi_{\epsilon}(\mathbf{x})$ has been widely called a kinetic potential [41-43]. Therefore, if $\nabla \cdot \gamma_{\epsilon}=0 \forall \epsilon$, then there is an orthogonality between $\nabla \varphi_{\epsilon}(\mathbf{x})$ and $\gamma_{\epsilon}(\mathbf{x})$ for all $\epsilon$. More generally, irrespective of $\nabla \cdot \gamma_{\epsilon}$ being zero or not, in the limit of $\epsilon \rightarrow 0, \varphi_{\epsilon}(\mathbf{x}) \rightarrow \varphi(\mathbf{x}), \gamma_{\epsilon} \rightarrow \gamma$, and $\gamma \cdot \nabla \varphi=0$.

$\gamma(\mathbf{x})=0$ is mathematically equivalent to detailed balance. Stochastic systems with $\gamma=0$ is widely considered as "nondriven" [45, 46] and is expected to approach to an equilibrium steady state in the long-time limit. For such systems, the free energy $F$ aquires additional meaning as the potential of Onsager's thermodynamics force, $\gamma_{\epsilon}=\mathbf{D}(\mathbf{x}) \nabla F$.

Collecting Eqs. (8), (9) and (10), we have a system of three equations

$$
\begin{gathered}
\mathbf{b}(\mathbf{x})=-\mathbf{D}(\mathbf{x}) \nabla \varphi(\mathbf{x})+\gamma(\mathbf{x}), \\
\nabla \varphi(\mathbf{x}) \cdot \gamma(\mathbf{x})=0, \\
\nabla \cdot(\omega(\mathbf{x}) \gamma(\mathbf{x}))=-\nabla \omega(\mathbf{x}) \cdot \mathbf{D}(\mathbf{x}) \nabla \varphi(\mathbf{x}),
\end{gathered}
$$

in which the vector fields $\mathbf{b}(\mathbf{x})$ and $\gamma(\mathbf{x})$ represent dynamics, $\mathbf{D}(\mathbf{x})$, which represents stochastic motion, can be thought as a geometric metrics, $\omega(\mathbf{x})$ represents local "measure" for the state space volume (degeneracy in the statistical mechanical termonology $2, \varphi(\mathbf{x})$ and $\ln \omega(\mathbf{x})$ are thermodynamic quantities akin to energy and entropy, respectively. The "noise structure" $\mathbf{D}(\mathrm{x})$ provides a unique geometry for the dynamics.

In the simplest case, if $\mathbf{D}(\mathbf{x})$ is the identity matrix, and $\omega(\mathbf{x})=1$ is the Lebesgue measure, then the equations in Eq. (12) become

$$
\begin{gathered}
\mathbf{b}(\mathbf{x})=-\nabla \varphi(\mathbf{x})+\gamma(\mathbf{x}), \\
\gamma(\mathbf{x}) \cdot \nabla \varphi(\mathbf{x})=0, \\
\nabla \cdot \gamma(\mathbf{x})=0
\end{gathered}
$$

The vector field $\gamma(\mathbf{x})$ is now volume preserving, and the system $\mathbf{x}^{\prime}(t)=\gamma(\mathbf{x})$ also has a conserved quantity $\varphi(\mathbf{x})$. System with Eq. (13) is intimately related to the classical Hamiltonian systems [47]. One of the most important features of this class of dynamics is that the $\varphi(\mathbf{x})$ gives the steady state probability distribution exactly for any finite $\epsilon$ in the form of $\pi_{\epsilon}(\mathbf{x}) \propto e^{-\varphi(\mathbf{x}) / \epsilon}$ according to Boltzmann's law, if one identifies $\epsilon$ with temperature [10, 25].

$\varphi$-based statistical mechanics and ensemble change. - It is seen immediately that if one computes a partition function from energy function $\varphi(\mathbf{x})$ and degeneracy $\omega(\mathbf{x})$ :

$$
Z(\epsilon)=\int_{\mathbb{R}^{n}} \omega(\mathbf{x}) e^{-\varphi(\mathbf{x}) / \epsilon} \mathrm{d} \mathbf{x},
$$

then $Z^{-1}(\epsilon) \omega(\mathbf{x}) e^{-\varphi(\mathbf{x}) / \epsilon}$ is the asymptotic probability density for $\pi_{\epsilon}(\mathbf{x})$. If the principle of equal probability is valid, e.g., $\nabla \cdot \gamma(\mathbf{x})=0$, then it is the stationary probability density of Eq. (1) for all $\epsilon>0$.

Let us now consider a bivariate stochastic dynamics with $\mathbf{x}$ and $y$ which is assumed to be a scalar for simplicity. The stationary joint probability for $\mathbf{x}$ and $y, p_{\epsilon}(\mathbf{x}, y)$ is related to the stationary conditional probability $p_{\epsilon}(\mathbf{x} \mid y)$ through the marginal distribution for variable $y, p_{\epsilon, y}(y): p_{\epsilon}(\mathbf{x}, y)=p_{\epsilon}(\mathbf{x} \mid y) p_{\epsilon, y}(y)$. In the asmptotic limit of $\epsilon \rightarrow 0$, this yields

$$
\varphi(\mathbf{x}, y)=\varphi(\mathbf{x} \mid y)+\varphi_{y}(y)
$$

\footnotetext{
${ }^{2}$ Actually, there is also a time-dependent equation for $\omega^{\mathrm{td}}(\mathbf{x}, t)$ : $\partial \omega^{\mathrm{td}}(\mathbf{x}, t) / \partial t=-\left(\mathbf{D}(\mathbf{x}) \nabla \varphi^{\mathrm{td}}(\mathbf{x}, t)\right) \cdot \nabla \omega^{\operatorname{td}}(\mathbf{x}, t)-\nabla$. $\left(\gamma^{\mathrm{td}}(\mathbf{x}, t) \omega^{\mathrm{td}}(\mathbf{x}, t)\right)$. The asymptotic expansion to this next-order also provides a natural viscosity solution for the HJE with a diffusion term $\epsilon \nabla \cdot\left(\omega^{\mathrm{td}}(\mathbf{x}, 0) \mathbf{D}(\mathbf{x}) \nabla \varphi^{\mathrm{td}}(\mathbf{x}, t)\right)$.
} 
and the partition functions

$$
Z_{\mathbf{x}, y}(\epsilon)=\int_{y} Z_{\mathbf{x} \mid y}(\epsilon ; y) e^{-\varphi_{y}(y) / \epsilon} \mathrm{d} y
$$

The $Z_{\mathbf{x} \mid y}$ is the partition function with fixed $y$, treated as a parameter, and $Z_{\mathbf{x}, y}$ is the partition function with fluctuating $y$. To asymptotically evaluating the integral in Eq. (16), it can be shown that at $y=\bar{y}$, the mean value of the fluctuating $y$ :

$$
\frac{\partial}{\partial y} \varphi_{y}(\bar{y})=\epsilon\left[\frac{\partial}{\partial y} \ln Z_{\mathbf{x} \mid y}(\epsilon ; y)\right]_{y=\bar{y}} \equiv \xi_{y}
$$

where $\xi_{y}$ is the conjugate variable to $y$. Therefore, the integrand in Eq. (16) can be approximately expressed as

$$
\varphi_{y}(y) \simeq \varphi_{y}(\bar{y})+\xi_{y}(y-\bar{y}) .
$$

Eqs. (16), (17), and (18) constitute J. W. Gibbs' theory of ensemble change. In doing so, the thermodynamics of a stationary system with fluctuating $y$ and the thermodynamics of a stationary system with fixed $y$ are logically connected via the large deviation theory. This derivation shares the same spirit as Helmholtz and Boltzmann's 1884 mchanical theory of heat [48, 49]: Both extend the notion of energy from a system with a fixed "parameter" $y$ to an entire family of systems with different $y$ 's [50].

An instantaneous deterministic energy balance equation. - While the $\epsilon F\left[p_{\epsilon}(\mathbf{x}, t)\right] \rightarrow \varphi(\hat{\mathbf{x}}(t))$ as $\epsilon \rightarrow 0$ and $p_{\epsilon}(\mathbf{x}, t) \rightarrow \delta(\mathbf{x}-\hat{\mathbf{x}}(t))$, the free energy dissipation, housekeeping heat, and entropy production rates [14,17], also known as non-adiabatic, adiabatic, and total entropy production rates [16], become

$$
\begin{aligned}
\epsilon f_{\mathrm{d}}\left[p_{\epsilon}(\mathbf{x}, t)\right] & \rightarrow[\nabla \varphi(\mathbf{x}) \cdot \mathbf{D}(\mathbf{x}) \nabla \varphi(\mathbf{x})]_{\mathbf{x}=\hat{\mathbf{x}}(t)} \\
\epsilon Q_{\mathrm{hk}}\left[p_{\epsilon}(\mathbf{x}, t)\right] & \rightarrow\left[\gamma(\mathbf{x}) \cdot \mathbf{D}^{-1}(\mathbf{x}) \boldsymbol{\gamma}(\mathbf{x})\right]_{\mathbf{x}=\hat{\mathbf{x}}(t)} \\
\epsilon e_{\mathrm{p}}\left[p_{\epsilon}(\mathbf{x}, t)\right] & \rightarrow\left[\mathbf{b}(\mathbf{x}) \cdot \mathbf{D}^{-1}(\mathbf{x}) \mathbf{b}(\mathbf{x})\right]_{\mathbf{x}=\hat{\mathbf{x}}(t)}
\end{aligned}
$$

All three quantities are non-negative. They are linked through a Pythagorean-like equation, $\forall \mathbf{x} \in \mathbb{R}^{n}$ :

$$
\|\mathbf{D}(\mathbf{x}) \nabla \varphi(\mathbf{x})\|^{2}+\|\gamma(\mathbf{x})\|^{2}=\|\mathbf{b}(\mathbf{x})\|^{2}
$$

under the inner product $\langle\mathbf{u}, \mathbf{v}\rangle \equiv \mathbf{v} \mathbf{D}^{-1} \mathbf{u}$ and thus $\|\mathbf{u}\|^{2}=$ $\mathbf{u D}^{-1} \mathbf{u} 3^{3}$

In the zero-noise limit, the deterministic motion follows $\mathrm{d} \mathbf{x}(t) / \mathrm{d} t=\mathbf{b}(\mathbf{x})$; the balanace equation $\mathrm{d} F / \mathrm{d} t \equiv-f_{\mathrm{d}}=$

\footnotetext{
${ }^{3}$ This Pythagorean relation actually exists for stochastic $f_{\mathrm{d}}, Q_{\mathrm{hk}}, e_{\mathrm{p}}$ with finite $\epsilon$ if one defines inner product

$$
\langle\mathbf{u}(\mathbf{x}), \mathbf{v}(\mathbf{x})\rangle \equiv \epsilon^{-1} \int_{\mathbb{R}^{n}} \mathbf{u}(\mathbf{x}) \mathbf{D}^{-1}(\mathbf{x}) \mathbf{v}(\mathbf{x}) p_{\epsilon}(\mathbf{x}, t) \mathrm{d} \mathbf{x},
$$

and identifies $f_{\mathrm{d}}=\left\|\epsilon \mathbf{D}(\mathbf{x}) \nabla \ln \left(p_{\epsilon}(\mathbf{x}, t) / \pi_{\epsilon}(\mathbf{x})\right)\right\|^{2}, Q_{\mathrm{hk}}=\| \mathbf{b}(\mathbf{x})-$ $\epsilon \mathbf{D}(\mathbf{x}) \nabla \ln \pi_{\epsilon}(\mathbf{x}) \|^{2}$, and $e_{\mathrm{p}}=\left\|\mathbf{b}(\mathbf{x})-\epsilon \mathbf{D}(\mathbf{x}) \nabla \ln p_{\epsilon}(\mathbf{x}, t)\right\|^{2}$.
}

$Q_{\mathrm{hk}}-e_{\mathrm{p}}$ now becomes

$$
\begin{aligned}
& \frac{\mathrm{d}}{\mathrm{d} t} \varphi(\mathbf{x}(t))=\mathbf{b}(\mathbf{x}) \cdot \nabla \varphi(\mathbf{x}) \\
= & \underbrace{\gamma(\mathbf{x}) \cdot \mathbf{D}^{-1}(\mathbf{x}) \gamma(\mathbf{x})}_{\text {non-conservative pump }}-\underbrace{\mathbf{b}(\mathbf{x}) \cdot \mathbf{D}^{-1}(\mathbf{x}) \mathbf{b}(\mathbf{x})}_{\text {energy dissipation }} .
\end{aligned}
$$

Both terms before and after the minus sign in Eq. (21) are nonnegative. Eq. (21) constitutes a deterministic, instantaneous energy balance law with the non-conservative pump as the source and energy dissipation as the sink, respectively. This result provides a rigorous notion of "energy" for complex dynamics with a stochastic kinematic description. For a classical mechanical system with potential force and friction, $\varphi$ is the sum of kinetic energy and potential energy, and the energy dissipation is due to the friction.

Perturbation theory of random processes and the higherorder approximation of $e_{\mathrm{p}}$ - - From Eq. (19), we have shown that the entropy production rate $e_{\mathrm{p}}$ is "extensive", i.e., $O\left(\epsilon^{-1}\right)$, and derived it's leading order as $\epsilon \rightarrow 0$. This leading order constitutes the part of entropy produced from the deterministic path of the "dissipative dynamics" $\hat{\mathbf{x}}^{\prime}(t)=\mathbf{b}(\hat{\mathbf{x}})$. We can further obtain higher-order terms of the entropy production rate, which yields the part of entropy production around the deterministic trajectory due to the infinitesimal fluctuation. We can visualize this by imaging that we follow the deterministic trajectory and measure the entropy production with a zoomedin scale. J. Keizer first realized this perspective provides a flucutation-dissipation theorem beyond equilibrium [51,52].

From a mathematical standpoint, the dissipation part of entropy production is on the scale of the law of large number, while the fluctuation part is on the scale for the central limit theorem. This suggests us that the latter can be obtained by the perturbation of random processes with an appropriate scale. Introducing $\mathbf{Z}_{\epsilon}(t)=\frac{1}{\sqrt{\epsilon}}\left(\mathbf{X}_{\epsilon}(t)-\hat{\mathbf{x}}(t)\right)$ and following the usual perturbation approach [30] with expansion $\mathbf{Z}_{\epsilon}=\mathbf{Z}^{(0)}+\sqrt{\epsilon} \mathbf{Z}^{(1)}+\cdots+\sqrt{\epsilon}^{k} \mathbf{Z}^{(k)}+\cdots$, we can write down a stochastic differential equation (SDE) for the $\mathbf{Z}^{(0)}(t)$

$$
\mathrm{d} \mathbf{Z}^{(0)}(t)=\mathbf{A}(\hat{\mathbf{x}}(t)) \mathbf{Z}^{(0)}(t) \mathrm{d} t+[2 \mathbf{D}(\hat{\mathbf{x}}(t))]^{\frac{1}{2}} \mathrm{~d} \mathbf{B}(t),
$$

where $\mathbf{A}(\mathbf{x})$ is the Jacobian matrix of $\mathbf{b}(\mathbf{x})$. Eq. (22) is a time-inhomogeneous linear SDE which can be solved as $\mathbf{Z}^{(0)}(t) \sim \mathcal{N}(0, \boldsymbol{\Sigma})$, where $\mathcal{N}$ represents Gaussian distribution and the covariance matrix $\Sigma$ satisfies the equation

$$
\frac{\mathrm{d} \boldsymbol{\Sigma}(t)}{\mathrm{d} t}=\mathbf{A}(\hat{\mathbf{x}}(t)) \boldsymbol{\Sigma}+\boldsymbol{\Sigma} \mathbf{A}(\hat{\mathbf{x}}(t))^{T}+2 \mathbf{D}(\hat{\mathbf{x}}(t)) .
$$

This equation under more restricted consideration had been obtained in [9, 52]. In addition to the small noise expansion for the SDE, we can also expand the FPE of the scaled process $\mathbf{Z}_{\epsilon}$ :

$$
\hat{p}_{\epsilon}(\mathbf{z}, t)=\sum_{n=0}^{\infty} \hat{p}_{n}(\mathbf{z}, t) \sqrt{\epsilon}^{n}
$$

in which the $\hat{p}_{0}(\mathbf{z}, t)$ corresponds exactly to the probability distribution of $\mathbf{Z}^{(0)}(t) \sim \mathcal{N}(0, \Sigma)$ [9]. By the change of variable 
$p_{\epsilon}(\mathbf{x}, t)=\frac{1}{\sqrt{\epsilon}} \hat{p}_{\epsilon}(\mathbf{z}, t)$ and plugging it into the equation of entropy production rate in Eq. (3), we can obtain the higher-order approximation

$$
\begin{aligned}
\epsilon e_{\mathrm{p}}\left[p_{\epsilon}\right] & =\left[\mathbf{b}(\mathbf{x}) \cdot \mathbf{D}^{-1}(\mathbf{x}) \mathbf{b}(\mathbf{x})\right]_{\mathbf{x}=\hat{\mathbf{x}}(t)} \\
& +\epsilon\left[\operatorname{tr}(\mathbf{M}(\mathbf{x}))+2 \mathbf{b}(\mathbf{x}) \cdot \mathbf{D}(\mathbf{x})^{-1} \mathbf{m}\right]_{\mathbf{x}=\hat{\mathbf{x}}(t)}+o(\epsilon)
\end{aligned}
$$

where

$$
\begin{aligned}
\mathbf{M}(\mathbf{x}) & =\mathbf{D}(\mathbf{x}) \boldsymbol{\Sigma}^{-1}+2 \mathbf{A}(\mathbf{x})+\mathbf{A}(\mathbf{x})^{T} \mathbf{D}(\mathbf{x})^{-1} \mathbf{A}(\mathbf{x}) \mathbf{\Sigma} \\
& +\mathbf{b}(\mathbf{x}) \cdot \mathbf{D}(\mathbf{x})^{-1} \mathbf{H}(\mathbf{x}) \mathbf{\Sigma}
\end{aligned}
$$

Note that $\mathbf{H}(\mathbf{x})$ is a rank 3 tensor, for the vector $\mathbf{v}=$ $\mathbf{b}(\mathbf{x}) \cdot \mathbf{D}(\mathbf{x})^{-1}, \mathbf{v H}(\mathbf{x})=\sum_{i} v_{i} \mathbf{H}_{i}(\mathbf{x})$, in which $\mathbf{H}_{i}(\mathbf{x})$ is the Hessian matrix of $b_{i}(\mathbf{x}) ; \boldsymbol{\Sigma}$ follows Eq. (23), and $\mathbf{m}=$ $\int \mathbf{z} \hat{p}_{1}(\mathbf{z}, t) \mathrm{d} \mathbf{z}$. Therefore, the $\epsilon$ order of the entropy production has the form

$$
\underbrace{\operatorname{tr}(\mathbf{M}(\hat{\mathbf{x}}(t)))}_{\text {Gaussian fluctuation }}+\underbrace{2 \mathbf{b}(\hat{\mathbf{x}}(t)) \cdot \mathbf{D}(\hat{\mathbf{x}}(t))^{-1} \mathbf{m}}_{\text {non-Gaussian fluctuation }}
$$

where the first part involves $\boldsymbol{\Sigma}$ (the second moment with respect to the Gaussian distribution $\left.\hat{p}_{0}(\mathbf{z}, t)\right)$ and the second part involves $\mathbf{m}$ (the first moment with respect to the next order $\hat{p}_{1}(\mathbf{z}, t)$ in the expansion (24)). Interestingly, if $\mathbf{Z}_{\epsilon}(t)$ is a time-inhomogeneous Ornstein-Uhlenbeck process, then the non-Gaussian fluctuation part is always zero. On the other hand, since the non-Gaussian part is due to the nonlinearity of the vector filed $\mathbf{b}$, this part of entropy production rate in the higher order exists uniquely in nonlinear dynamics, e.g. in limit cycles.

Discussion. - In the theory of ordinary differential equations, Hamiltonian dynamics with the conserved $H$ functions and gradient systems with potential functions are two special classes that have been extensively studied [47]. For a general nonlinear dynamics $\dot{\mathbf{x}}=\mathbf{b}(\mathbf{x})$, it is not known whether it always has an associated "energetics". Ventsel and Freidlin's large deviation theory revealed that if $\mathbf{b}(\mathbf{x})$ is the zero-noise limit of a noisy dynamics, a global quasi-potential function $\varphi(\mathbf{x})$ exists [21-23]. In fact, $\mathbf{b}(\mathbf{x})=\mathbf{D}(\mathbf{x}) \nabla \varphi(\mathbf{x})+\gamma(\mathbf{x})$, where $\gamma(\mathbf{x}) \perp \nabla \varphi(\mathbf{x})$ at every $\mathbf{x}$. Graham and Tél further showed [24] $\nabla \cdot(\omega(\mathbf{x}) \gamma(\mathbf{x}))=-\nabla \omega(\mathbf{x}) \cdot \mathbf{D}(\mathbf{x}) \nabla \varphi(\mathbf{x})$, where $\omega(\mathbf{x})$ represents a proper local measure at $\mathbf{x}$. The present work shows that this system of dynamic equations has a thermodynamic interpretation via a geometric equation: $\|\mathbf{b}\|^{2}=$ $\|\mathbf{D} \nabla \varphi\|^{2}+\|\gamma\|^{2}$, corresponding to the instantaneous rates of total entropy production, free energy dissipation, and housekeep heat, respectively.

If one identifies $\dot{\mathbf{x}}=\mathbf{b}(\mathbf{x})$ as a kinematic description of a complex dynamics, then the system in Eq. (12) and Eq. (21) provide an energetic description that is hidden under the kinematics. A few words concerning the role of $\mathbf{D}(\mathbf{x})$ are in order. The concept of a gradient field on $\mathbb{R}^{n}$ requires a notion of distance. This is naturally provided by the noise structure embedded in $\mathbf{D}(\mathbf{x})$. This is precisely A. N. Kolmogorov's insights on the nature of probability theory: One needs to have a probability given before carrying out probabilistic computations.
For complex systems, not all "stochasticity" are due to thermal noises. In fact, the Mori-Zwanzig theory of projection operator clearly shows that [53] the dynamics of a projection necessarily induces, in general, a stochastic term and a nonMarkovian memory term.

When $\gamma=0$, the FPE in Eq. (1) is a gradient flow in a proper mathematical space [54]. In terms of the Pythagoreanlike equation in Eq. (20), the stochastic dynamics following the Eq. (1) with $\gamma=0$ has a maximal $f_{\mathrm{d}}$ : One leg of a "triangle" has the same length as the triangle's hypotenuse. Thus, under an appropriate geometry, Onsager's principle of maximum dissipation can be generalized to nonlinear regime [55]. In recent years, the theory of nonequilibrium landscape has gained wider recognitions in biological physics [56, 57, 59]. Since for a nonequilibrium stochastic system, its stationary state still has highly complex motions as NESS flux [45 46, 58], the $\varphi$ is only half the story: the $\gamma$ and its related $\omega$ provide the characterization of the NESS motion on each and every $\varphi$ level set.

We thank Jin Feng, Hao Ge, Yi-An Ma, Mark Peletier, and Jin Wang for helpful discussions, and an anonymous reviewer for feedback. This work was supported in part by NIH R01 grants GM109964 and GM135396 (both PI: Sui Huang), and the Olga Jung Wan Endowed Professorship for the first author.

\section{REFERENCES}

[1] H. Ge and H. Qian, Mesoscopic kinetic basis of macroscopic chemical thermodynamics: A mathematical theory, Phys. Rev. E 94, 052150 (2016).

[2] H. Ge and H. Qian, Mathematical formalism of nonequilibrium thermodynamics for nonlinear chemical reaction systems with general rate law, J. Stat. Phys. 166, 190 (2017).

[3] L. Onsager, Reciprocal relations in irreversible processes. I, Phys. Rev. 37, 405 (1931).

[4] J. G. Kirkwood, Statistical mechanics of fluid mixtures, J. Chem. Phys. 3, 300 (1935).

[5] T. L. Hill, An Introduction to Statistical Thermodynamics, (Dover, New York, 1986).

[6] A. I. Khinchin, Mathematical Foundations of Information Theory, R. A. Silverman and M. D. Friedman transl. (Dover, New York, 1957).

[7] M. C. Mackey, The dynamic origin of increasing entropy, Rev. Mod. Phys. 61, 981 (1989).

[8] P. W. Anderson, More is different: Broken symmetry and the nature of the hierarchical structure of science, Science 177, 393 (1972).

[9] C. Gardiner, Stochastic Methods: A Handbook for the Natural and Social Sciences, 4th ed. (Springer, New York, 2009).

[10] H. Qian, Thermodynamics of the general diffusion process: Equilibrium supercurrent and nonequilibrium driven circulation with dissipation, Eur. Phys. J. Sp. Top. 224, 781 (2015).

[11] P. G. Bergmann and J. L. Lebowitz, New approach to nonequilibrium processes, Phys. Rev. 99, 578 (1955).

[12] H. Qian, Relative entropy: Free energy associated with equilibrium fluctuations and nonequilibrium deviations, Phys. Rev. E 63, 042103 (2001). 
[13] T. M. Cover and J. A. Thomas, Elements of Information Theory, 2nd ed., Wiley, New York (2006).

[14] H. Ge and H. Qian, Physical origins of entropy production, free energy dissipation, and their mathematical representations, Phys. Rev. E 81, 051133 (2010).

[15] C. van den Broeck and M. Esposito, The three faces of the second law: II. Fokker-Planck formulation, Phys. Rev. E 82, 011144 (2010).

[16] M. Esposito and C. van den Broeck, Three detailed fluctuation theorems, Phys. Rev. Lett. 104, 090601 (2010).

[17] H. Qian, A decomposition of irreversible diffusion processes without detailed balance, J. Math. Phys. 54, 053302 (2013).

[18] C. Jarzynski, Equalities and inequalities: Irreversibility and the second law of thermodynamics at the nanoscale, Ann. Rev. Cond. Matt. Phys. 2, 329 (2011).

[19] U. Seifert, Stochastic thermodynamics, fluctuation theorems, and molecular machines, Rep. Prog. Phys. 75, 126001 (2012).

[20] C. van den Broeck and M. Esposito, Ensemble and trajectory thermodynamics: A brief introduction, Physica A 418, 6 (2015).

[21] A. D. Ventsel and M. I. Freidlin, On small random perturbations of dynamical systems, Russ. Math. Survey 25, 1 (1970).

[22] M. I. Dykman and M. A. Krivoglaz, Theory of fluctuational transitions between stable states of nonlinear oscillators, Sov. Phys. JETP 50, 30 (1979).

[23] R. Graham and T. Tél, Weak-noise limit of Fokker-Planck models and nondifferentiable potentials for dissipative dynamical systems, Phys. Rev. A, 31, 1109 (1985).

[24] R. Graham and T. Tél, On the weak-noise limit of Fokker-Planck models, J. Stat. Phys, 35, 729 (1984).

[25] H. Qian, The zeroth law of thermodynamics and volumepreserving conservative system in equilibrium with stochastic damping, Phys. Lett. A 378, 609 (2014).

[26] H. Ge and H. Qian, Landscapes of non-gradient dynamics without detailed balance: Stable limit cycles and multiple attractors, Chaos, 22, 023140 (2012).

[27] L.-S. Young, What are SRB measures, and which dynamical systems have them? J. Stat. Phys. 108, 733 (2002).

[28] Y.-A. Ma, Q. Tan, R. Yuan, B. Yuan and P. Ao, Potential function in a continuous dissipative chaotic system: Decomposition scheme and role of strange attractor, Int. J. Bifurcation Chaos 24, 1450015 (2014).

[29] R. Balian, From Microphysics to Macrophysics: Methods and Applications of Statistical Physics. Volume 1 (Springer-Verlag, Berlin Heidelberg, 2007)

[30] M. I. Freidlin and A. D. Wentzell, Random Perturbations of Dynamical Systems (Spinger-Verlag, New York, 1984).

[31] N. G. Van Kampen, Stochastic Processes in Physics and Chemistry, 3rd ed. (North Holland, 2007)

[32] U. C. Taubër, Critical dynamics: A Field theory approach to Equilibrium and Non-Equilibrium Scaling Behavior (Cambridge University Press, 2014)

[33] A. Lense and M. Laguës, Scale Invariance: From Phase Transitions to Turbulence, (Springer-Verlag, Berlin Heidelberg, 2012)

[34] T. G. Kurtz, The Relationship between Stochastic and Deterministic Models for Chemical Reactions, J. Chem. Phys. 57, 2976 (1972)

[35] A. Dembo and O. Zeitouni, Large Deviations Techniques and Applications, 2nd ed. (Springer, New York, 1998).

[36] Y. Oono, Large deviation and statistical physics, Progr. Theoret. Phys. Supp. 99, 165 (1989).

[37] $\mathrm{H}$. Touchette, The large deviation approach to statistical mechanics, Phys. Rep. 478, 1 (2009).
[38] E. Smith, Large-deviation principles, stochastic effective actions, path entropies, and the structure and meaning of thermodynamic description, Rep. Prog. Phys. 74, 046601 (2011).

[39] H. Ge and H. Qian, Analytical mechanics in stochastic dynamics: Most probable path, large-deviation rate function and Hamilton-Jacobi equation, Int. J. Mod. Phys. B 26, 1230012 (2012).

[40] H. Qian, Mesoscopic nonequilibrium thermodynamics of single macromolecules and dynamic entropy-energy compensation, Phys. Rev. E 65, 016102 (2002).

[41] R. Graham and H. Haken, Generalized thermodynamic potential for Markoff systems in detailed balance and far from thermal equilibrium, Z. Phys. 243, 289 (1971).

[42] R. Kubo, K. Matsuo and K. Kitahara, Fluctuation and relaxation of macrovariables, J. Stat. Phys. 9, 51 (1973).

[43] G. Nicolis and R. Lefever, Comment on the kinetic potential and the Maxwell construction in non-equilibrium chemical phase transitions, Phys. Lett. A 62, 469 (1977).

[44] H. Qian, M. Qian and X. Tang, Thermodynamics of the general diffusion process: Time-reversibility and entropy production, J. Stat. Phys. 107, 1129 (2002).

[45] X.-J. Zhang, H. Qian and M. Qian, Stochastic theory of nonequilibrium steady states and its applications (Part I), Phys. Rep. 510, 1 (2012).

[46] H. Ge, M. Qian and H. Qian, Stochastic theory of nonequilibrium steady states (Part II): Applications in chemical biophysics, Phys. Rep. 510, 87 (2012).

[47] L. Perko, Differential Equations and Dynamical Systems, 3rd ed. (Springer, New York, 2001).

[48] G. Gallavotti, Statistical Mechanics: A Short Treatise (Springer, Berlin, 1999).

[49] M. Campisi, On the mechanical foundations of thermodynamics: the generalized Helmholtz theorem, Stud. History Philos. Mod. Phys. 36, 275 (2005).

[50] Y.-A. Ma and H. Qian, A thermodynamic theory of ecology: Helmholtz theorem for Lotka-Volterra equation, extended conservation law, and stochastic predator-prey dynamics, Proc. R. Soc. A 471, 20150456 (2015).

[51] J. Keizer, Nonequilibrium thermodynamics and stability of states far from equilibrium, Acc. Chem. Res. 12, 243 (1979).

[52] J. Keizer, Statistical Thermodynamics of Nonequilibrium Processes (Springer-Verlag, New York, 1987)

[53] G. D. J. Phillies, Elementary Lectures in Statistical Mechanics (Springer, New York, 2000).

[54] A. Mielke, M. A. Peletier and M. Renger, On the relation between gradient flows and the large-deviation principle, with applications to Markov chains and diffusion, Potential Anal. 41, 1293 (2014).

[55] A. Mielke, M. A. Peletier and M. Renger, A generalization of Onsager's reciprocity relations to gradient flows with nonlinear mobility, J. Non-Equil. Thermody. 41, 141 (2016).

[56] P. Ao, Emerging of stochastic dynamical equalities and steady state thermodynamics from Darwinian dynamics, Comm. Theoret. Phys. 49, 1073 (2008).

[57] H. Qian and H. Ge, Mesoscopic biochemical basis of isogenetic inheritance and canalization: Stochasticity, nonlinearity, and emergent landscape, MCB: Mol. Cell. Biomech. 9, 1 (2012).

[58] J. Wang, Landscape and flux theory of non-equilibrium dynamical systems with application to biology, Adv. Phys. 64, 1 (2015).

[59] S. Huang, F. Li, J. X. Zhou and H. Qian, Processes on the emergent landscapes of biochemical reaction networks and heterogeneous cell population dynamics: Differentiations in living mat- 
ters, J. R. Soc. Interf. 14, 20170097 (2017). 